\title{
Modified Lorentz transformations and quantum mechanics
}

Jae-Kwang Hwang ( $\nabla$ jkhwang.koh@gmail.com )

JJJ Physics Laboratory https://orcid.org/0000-0002-4100-3473

\section{Research Article}

Keywords: Modified Lorentz transformations, 4-D Euclidean space, Quantum mechanics, Wave function collapse, Absolute time, Space expansion.

Posted Date: February 26th, 2021

DOl: https://doi.org/10.21203/rs.3.rs-278147/v1

License: (9) This work is licensed under a Creative Commons Attribution 4.0 International License. Read Full License 


\title{
Modified Lorentz transformations and quantum mechanics
}

\author{
Jae-Kwang Hwang \\ JJJ Physics Laboratory, Brentwood, TN 37027 USA \\ e-mail: jkhwang.koh@gmail.com
}

\begin{abstract}
;
We live in the 4-D Euclidean space. The $4^{\text {th }}$ dimension is assigned as the absolute time (ct) axis and energy axis $\left(\mathrm{cP}_{\mathrm{t}}=\mathrm{E}_{0}\right)$ based on 4-dimensional Euclidean space. This $4^{\text {th }}$ dimension can be indirectly felt through the observable relative time $\left(\mathrm{ct}_{1}\right)$ and observable total energy $\left(\mathrm{cP}_{\mathrm{tl}}=\mathrm{E}\right)$. The space-time distance is $\mathrm{d}(\mathrm{x} 1 \times 2 \times 3 \times 4)=\mathrm{ct}$. The modified Lorentz transformations are introduced by the time-matching of the absolute times in the 4-D Euclidean space. The size of $x^{\prime}$ ( or $\Delta x^{\prime}$ ) of the moving object is expanded to the size of $x=\gamma x^{\prime}$ ( or $\Delta x=\gamma \Delta x$ '). These modified Lorentz transformations are approximated to the Lorentz transformations as $\mathrm{t} \rightarrow \mathrm{t}_{\mathrm{l}}$ when $\mathrm{v} / \mathrm{c}<<1$ and to the Galilean transformations as v/c is close to zero. The relative time $\left(\mathrm{t}_{1}\right)$ and energy $(\mathrm{E})$ are defined as the 4-dimensional distance and 4-dimensional volume, respectively. The geometrical space-time shape has the (x1,x2,x3,ct) coordinate system with the metric signature of $(++++)$ but not the $\left(\mathrm{x} 1, \mathrm{x} 2, \mathrm{x} 3, \mathrm{ct}_{1}\right)$ coordinate system with the metric signature of $(+--)$. Therefore, $\mathrm{d}(\mathrm{x} 1 \mathrm{x} 2 \mathrm{x} 3 \mathrm{x} 4)^{2}=\left(\mathrm{ct}_{1}\right)^{2}=(\mathrm{ct})^{2}+\mathrm{x}^{2}=\mathrm{x} 1^{2}+\mathrm{x} 2^{2}+\mathrm{x} 3^{2}$ $+\mathrm{x} 4^{2}$ and $\mathrm{V}(\mathrm{x} 1 \mathrm{x} 2 \mathrm{x} 3 \mathrm{x} 4)=\mathrm{E}=\mathrm{mc}^{2}=\Delta(\mathrm{ct}) \Delta \mathrm{x} 1 \Delta \mathrm{x} 2 \Delta \mathrm{x} 3$ from $(\mathrm{x} 1, \mathrm{x} 2, \mathrm{x} 3, \mathrm{x} 4)$ of the geometrical spacetime shape. The warped shape can be described as the wave function of the quantum mechanics. The instant force action, twin paradox and possible space travel are explained by the absolute time and wave function collapse of the modified Lorentz transformations and quantum mechanics.
\end{abstract}

Key words; Modified Lorentz transformations, 4-D Euclidean space, Quantum mechanics, Wave function collapse, Absolute time, Space expansion.

\section{Introduction}

The space-time geometry has been one of the main topics of the physics research. This research includes the special and general relativities, quantum mechanics, manifold mathematical physics, cosmology and standard model [1-14]. The Lorentz transformations have been derived in the special relativity [1,2]. The $4^{\text {th }}$ dimension in the 4-D Minkowski space has been considered as the observable time dimension which is separated from the rest three space dimensions. In the physics research, the observed time of $t$ (expressed as $t_{1}$ in the present paper in Table 1) has been treated as the real relative time axis in the space-time geometry of 4-D Minkowski space. Because of this definition, the space-time geometry has been very complicated as seen in the special and general relativities. In this case, the space-time geometry has been expressed as the coordinates of $\left(\mathrm{x} 1, \mathrm{x} 2, \mathrm{x} 3, \mathrm{ct}_{1}\right)$ with the metric signature of $(+---)[1-3,10,14]$. In this case, the space-time distance is $\mathrm{s}^{2}=\left(\mathrm{ct}_{1}\right)^{2}-\mathrm{x} 1^{2}-\mathrm{x} 2^{2}-\mathrm{x} 3^{2}$. However, in the present work, The space-time geometric shape has the $(\mathrm{x} 1, \mathrm{x} 2, \mathrm{x} 3, \mathrm{ct})$ coordinate system with the metric signature of $(++++)$ based on the 4-D Euclidean space. Therefore, the space-time distance is $\mathrm{d}(\mathrm{x} 1 \mathrm{x} 2 \mathrm{x} 3 \mathrm{x} 4)^{2}=(\mathrm{ct})^{2}=(\mathrm{ct})^{2}+\mathrm{x}^{2}=\mathrm{x} 1^{2}+$ $x 2^{2}+x 3^{2}+x 4^{2}$. The time of $t$ in the special relativity based on 4-D Minkowski space is expressed as the relative time of $t_{1}$ in the Modified Lorentz transformations based on the 4-D Euclidean space of the present paper. Because this research includes the new physical concepts on the modified Lorentz transformations, the results of the present work are presented in the introduction section 
as shown in Figs. 1 - 3 and Tables 1 and 2 for the readers. In the present work, the observable relative time of $\mathrm{ct}_{1}$ is defined as the space-time distance as shown in Figs. 1 - 3. In this case, the

Table 1. Comparison of Modified Lorentz transformations (MLT), Lorentz transformations (LT) and Galilean transformations (GT). The times of $t_{1}$ and $t$ are called as the relative time and absolute time, respectively.

\begin{tabular}{|c|c|c|}
\hline $\begin{array}{l}\text { Modified Lorentz } \\
\text { transformations }\end{array}$ & $\begin{array}{l}\text { Lorentz } \\
\text { transformations }\end{array}$ & $\begin{array}{l}\text { Galilean } \\
\text { transformations }\end{array}$ \\
\hline $\begin{array}{l}\text { By using } \\
x=c t, \text { and } x^{\prime}=c t^{\prime} \\
t_{1}=\gamma t, \text { and } t_{1}^{\prime}=\gamma t^{\prime} \\
t^{\prime}=\gamma\left(t-v x / c^{2}\right) \\
x^{\prime}=\gamma(x-v t)\end{array}$ & $\begin{aligned} v / c & <<1 \\
t & \rightarrow t_{1}, t^{\prime} \rightarrow t_{1}^{\prime} \\
t_{1}^{\prime} & =\gamma\left(t_{1}-v x / c^{2}\right) \\
x^{\prime} & =\gamma\left(x-v t_{1}\right)\end{aligned}$ & $\begin{array}{l}v / c \rightarrow 0 \\
t_{1}^{\prime}=t_{1} \\
x^{\prime}=x-v t_{1}\end{array}$ \\
\hline $\begin{array}{l}\mathrm{t}^{\prime}=\gamma\left(\mathrm{t}-\mathrm{vx} / \mathrm{c}^{2}\right)= \\
\gamma\left(\left(1-\mathrm{v}^{2} / \mathrm{c}^{2}\right)^{0.5} \mathrm{t}_{\mathrm{I}}-\mathrm{vx} / \mathrm{c}^{2}\right)\end{array}$ & $t_{1}^{\prime}=\gamma\left(t_{1}-v x / c^{2}\right)$ & $t_{1}^{\prime}=t_{1}$ \\
\hline $\begin{array}{l}x^{\prime}=\gamma(x-v t)= \\
\gamma\left(x-v\left(1-v^{2} / c^{2}\right)^{0.5} t_{I}\right)\end{array}$ & $x^{\prime}=\gamma\left(x-v t_{1}\right)$ & $x^{\prime}=x-v t_{1}$ \\
\hline
\end{tabular}

Table 2. Comparison between the quantum mechanics and general relativity theory.

\begin{tabular}{|c|c|c|c|}
\hline $\begin{array}{c}\text { Dimen- } \\
\text { sions } \\
\text { (n) }\end{array}$ & & Volume & $\begin{array}{l}\text { Distance } \\
(\Delta \ell)\end{array}$ \\
\hline 4 & Energy & $\begin{array}{l}E=c \Delta t \Delta V \\
=c \Delta t \Delta x 1 \Delta x 2 \Delta x 3 \\
=\int c t(x) d x \\
=\int E|\Psi(x)|^{2} d x \\
\text { (Quantum mechanics) }\end{array}$ & 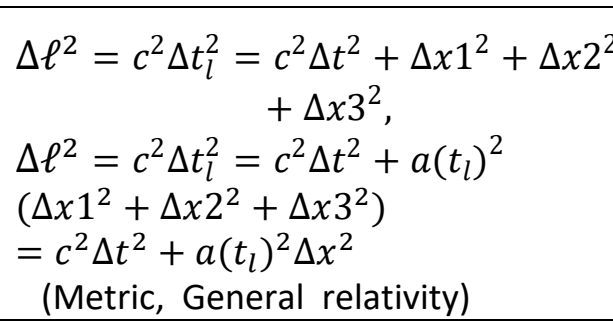 \\
\hline 3 & Volume & $V=\Delta x 1 \Delta x 2 \Delta x 3$ & $\begin{array}{c}\Delta \ell^{2}= \\
\Delta x 1^{2}+\Delta x 2^{2}+\Delta x 3^{2}\end{array}$ \\
\hline 2 & Area & $A=\Delta x 1 \Delta x 2$ & $\begin{array}{c}\Delta \ell^{2}= \\
\Delta x 1^{2}+\Delta x 2^{2}\end{array}$ \\
\hline 1 & Length & $l=\Delta x 1$ & $\Delta \ell^{2}=\Delta x 1^{2}$ \\
\hline
\end{tabular}

space-time geometry is expressed as the coordinates of $(\mathrm{x} 1, \mathrm{x} 2, \mathrm{x} 3, \mathrm{ct})$ with the metric signature of $(++++)$. This new geometry has been applied to the momentum space, too. Also, the modified Lorentz transformations are, for the first time, introduced in the coordinates of $(\mathrm{x} 1, \mathrm{x} 2, \mathrm{x} 3, \mathrm{ct})$ in Figs. 2 and 3 and Tables 1 and 2. Then it can be reasonably applied to all cases of the general relativity, quantum mechanics, manifold mathematical physics, cosmology and standard model including the CPT symmetric universe, big-bang process and special and general relativities $[5,6,12,13]$. 
First, the time, $\mathrm{t}$ in the Lorentz transformations (LT) of the special relativity corresponds to $\mathrm{t}_{1}$ in the modified Lorentz transformations (MLT) in Table 1. The photon with the constant speed (c) is the flat space with the zero charge [15]. The particle and matter with non-zero rest mass (mo) are the warped spaces. The rest mass energy of $\mathrm{E}_{0}=\mathrm{m}_{0} \mathrm{c}^{2}$ is defined as the four-dimension space volume of $\Delta(\mathrm{ct}) \Delta \mathrm{x} 1 \Delta \mathrm{x} 2 \Delta \mathrm{x} 3$ in Table 2 and Fig. 4 . The whole flat space (photon) can be treated like a rest particle because a particle size with the light velocity (c) is infinite in MLT as shown in the present work. Then $\frac{x}{x^{\prime}} \rightarrow \infty$ as $\mathrm{v} \rightarrow \mathrm{c}$ in MLT. Note that the negative time, negative mass (energy) and negative momenta are possible in Fig. 1 [15]. E $0<0$ and $\mathrm{m}_{0}<0$ for $\mathrm{p}_{\mathrm{t}}<0$. The wellknown Lorentz transformations in the special relativity are, for the first time, modified by using the 4-dimensional Euclidean coordinates. The $4^{\text {th }}$ dimension axis is the absolute time (ct) axis in the present work. Then the modified Lorentz transformations are written about this absolute time (ct) rather than the observable relative time $\left(\mathrm{ct}_{1}\right)$. Then the metric signature of $(+,+,+,+)$ is used rather than that of $(+,-,-,-)$. If this idea is reasonable enough to be considered, this work could extend the research on the general relativity and standard model. I wish this can open the gate for the related research in the near future.

2. Modified Lorentz transformations and new coordinates of $(\mathrm{x} 1, \mathrm{x} 2, \mathrm{x} 3, \mathrm{ct})$

The $4^{\text {th }}$ space dimension axis is the same as the absolute time axis of ct in Figs $1-4[15,16]$. The 4-dimensional $4 \times 4$ matrix is the simple extension of the 3 -dimensional $3 \times 3$ matrix. Therefore, $c \Delta \mathrm{t}_{1}$ is defined as the space-time distance of $\mathrm{d}$ which is treated as the observable relative time of $\mathrm{t}_{1}=$ $\mathrm{d} / \mathrm{c}$. The real absolute time of $\mathrm{t}$ is along the $4^{\text {th }}$ dimension axis. The 4-dimensional space geometry can be described as coordinates of $(\mathrm{x} 1, \mathrm{x} 2, \mathrm{x} 3, \mathrm{x} 4)=(\mathrm{x} 1, \mathrm{x} 2, \mathrm{x} 3, \mathrm{ct})$ with the metric signature of $(++$

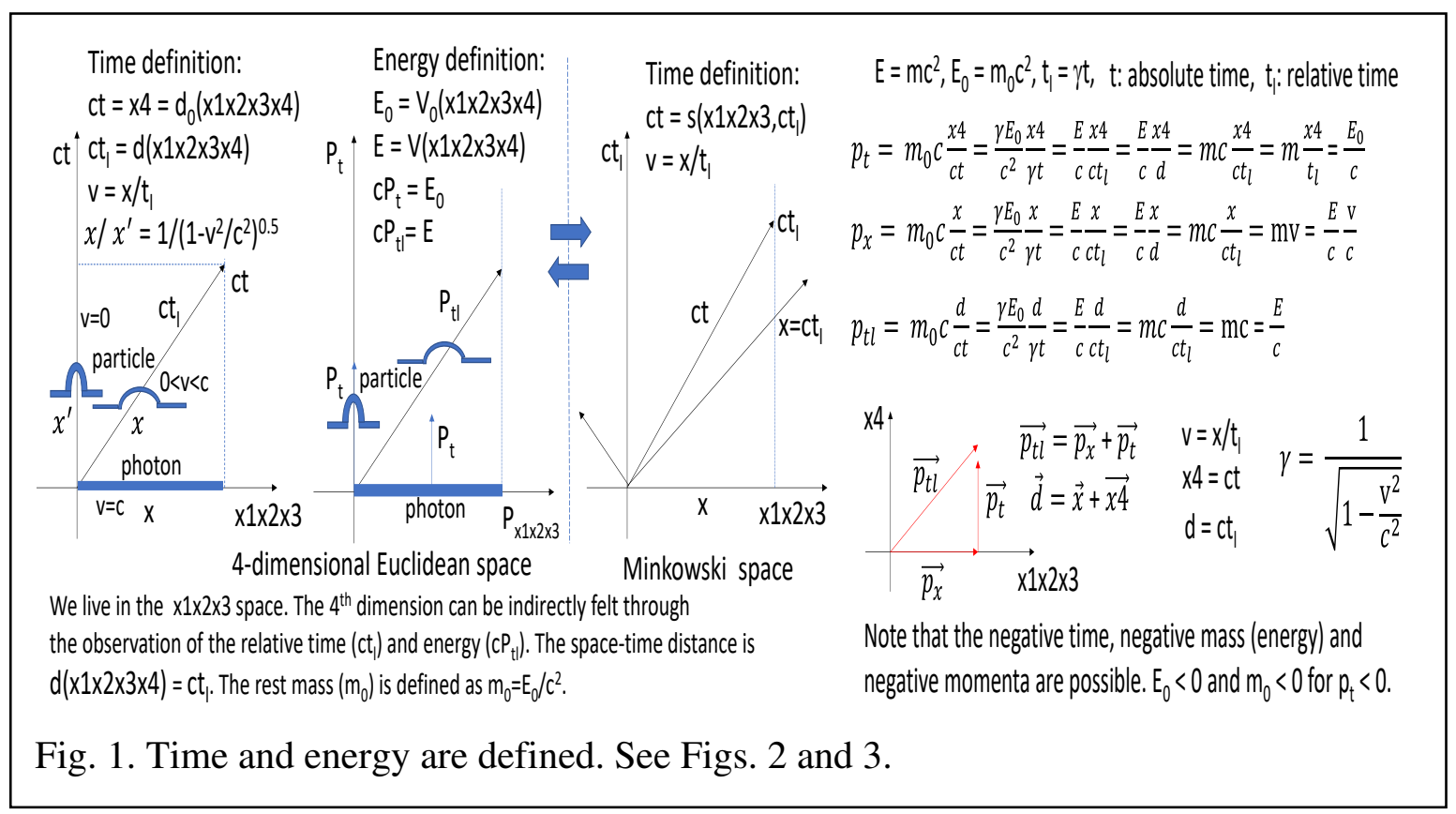

++ ). The 4-dimensional vectors can be added and subtracted in the coordinate system of $(\mathrm{x} 1, \mathrm{x} 2, \mathrm{x} 3, \mathrm{x} 4)=(\mathrm{x} 1, \mathrm{x} 2, \mathrm{x} 3, \mathrm{ct})$ with the metric signature of $(++++)$. In this coordinate system, the space-time distance of $\mathrm{d}$ for one 4-dimensional vector is generally preserved when the 4dimensional vectors are added and subtracted. 
When the primed coordinate system is moving with the velocity of $\mathrm{v}$ along the $\mathrm{x}$-axis of the unprimed coordinate system, the Galilean transformation is $\mathrm{x}^{\prime}=\mathrm{x}-\mathrm{vt}_{1}$ and $\mathrm{t}_{\mathrm{l}}{ }^{\prime}=\mathrm{t}_{\mathrm{l}}$. The Galilean transformations are good as the low-speed approximation of $\mathrm{v} / \mathrm{c} \rightarrow 0$ in Table 1 . Then, the Lorentz transformations are previously known as $\mathrm{x}^{\prime}=\gamma\left(\mathrm{x}-\mathrm{vt}_{\mathrm{l}}\right)$ and $\mathrm{t}_{\mathrm{l}}{ }^{\prime}=\gamma\left(\mathrm{t}_{1}-\mathrm{vx} / \mathrm{c}^{2}\right)$ by considering the photon speed of $\mathrm{c}$ and the metric signature of $(+---)$. Here $\gamma=1 /\left(1-\mathrm{v}^{2} / \mathrm{c}^{2}\right)^{0.5}$. The relative time of $t_{1}$ in these Lorentz transformations with the metric signature of (+ - - ) in the special relativity corresponds to the observable relative time of $t_{1}$ in the present work with the metric signature of $(+$ +++ ) in the modified Lorentz transformations in Figs. $1-4$. Therefore, these modified Lorentz transformations can be made by considering two times of $t$ and $t_{1}$ in terms of the 4-dimensional

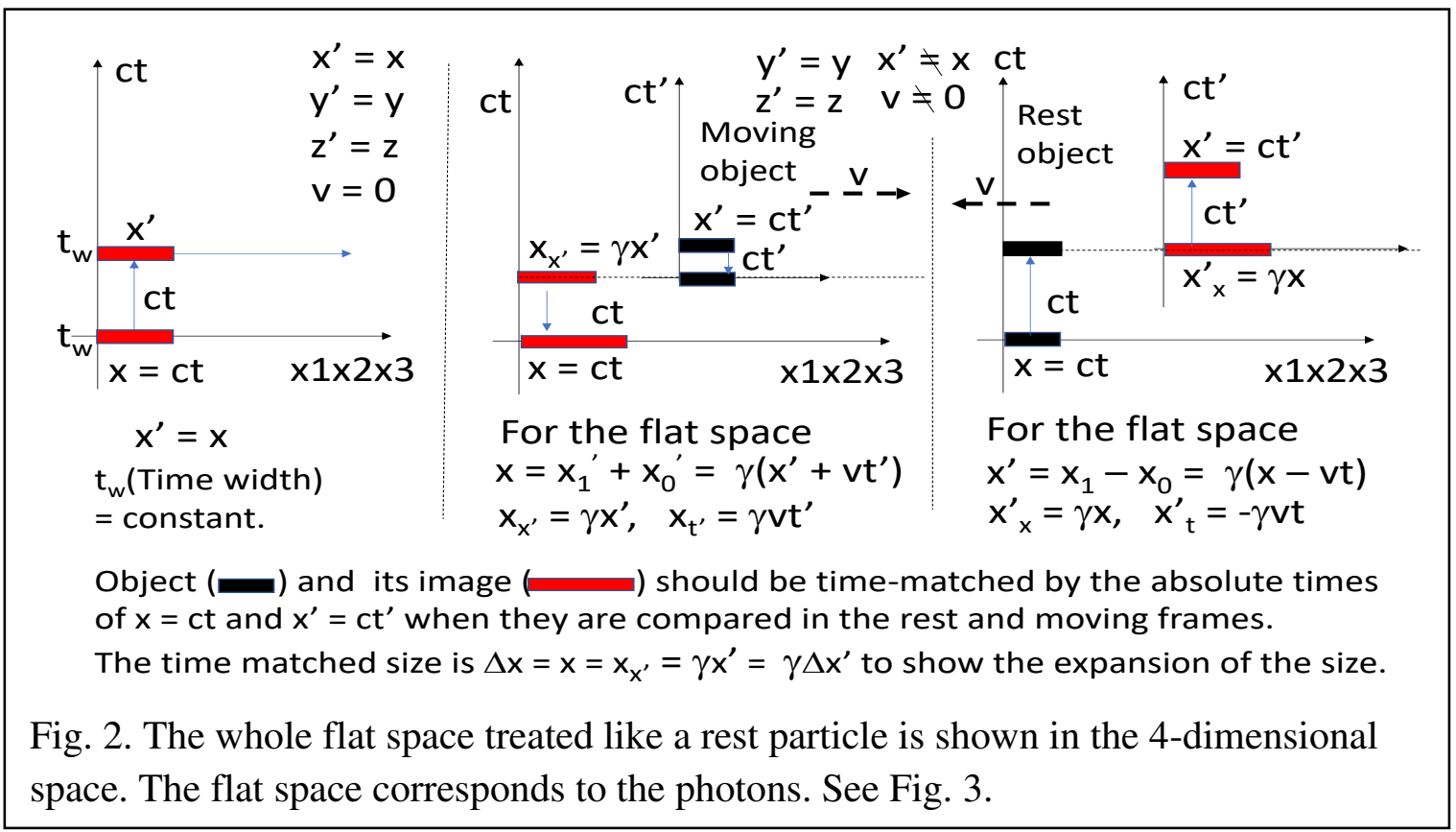

$\mathrm{x} 1 \times 2 \times 3 \times 4$ space (4-D Euclidean space) in Figs. 1 - 4. The observable relative time $\left(\mathrm{t}_{\mathrm{l}}\right)$ and real absolute time $(\mathrm{t})$ should be separately considered. In Figs $1-4$ and Table $1, t=t_{1} / \gamma$. Therefore, $t \rightarrow$ ti only when $\mathrm{v} / \mathrm{c}<<0$.

So, I tried to derive the modified Lorentz transformations in the 4-dimensional $\mathrm{x} 1 \mathrm{x} 2 \mathrm{x} 3 \mathrm{x} 4$ space by using the relation of $\mathrm{t}_{1}=\gamma \mathrm{t}$. It is assumed that the $\mathrm{x} 1, \mathrm{x} 2$ and $\mathrm{x} 3$ axes correspond to the $\mathrm{x}, \mathrm{y}$ and $\mathrm{z}$ axes in Figs. 2 and 3. For the flat space like photons, $\Delta x=c \Delta t_{1}$. In Figs. 2 and 3, the relative velocity ( $\mathrm{v}$ ) of two 4-dimensional spaces is not zero along the $\mathrm{x}$ axis. Then $\mathrm{v}=\mathrm{x}_{0} / \mathrm{t}_{\mathrm{l}}$. And the modified Lorentz transformations are derived by using the relation of $t_{1}=\gamma t$. In Fig. 3, how to derive the modified Lorentz transformations is shown. Then the observable relative time of $t_{1}$ in the previous Lorentz transformations (LT) in the special relativity is replaced with the real absolute time of $t$ in the new modified Lorentz transformations (MLT). This means that the previous Lorentz transformations (LT) are the approximate form of the new modified Lorentz transformations under the assumption that $\mathrm{t} \rightarrow \mathrm{t}_{1}$ when $\mathrm{v} / \mathrm{c}<<1$ in Table 1 . In the new modified Lorentz transformations, by using $x=c t$ and $x^{\prime}=c t^{\prime}$ in Fig. 3, $x^{\prime}=\gamma(1-v / c) x$ and $t^{\prime}=\gamma(1-v / c) t$. In Table 1, Lorentz transformations and Galilean transformations are compared for the better explanation. In Table 2, the energy and space-time distance are, for the first time, defined. 
The space size of $\mathrm{x}$ in the modified Lorentz transformations of Figs. 2 and 3 are generally expressed. When the object is moving in the moving primed frame, the object size is X' seen in the primed moving frame. This object size is expanded to $\mathrm{x}$ seen in the unprimed rest frame. Therefore, generally $x=\gamma\left(x^{\prime}+v t^{\prime}\right)$ in Fig. 3. In this case, the object in the moving frame and its image in the rest frame are not time-matched for the absolute times. It is fine to use these expressions for the modified Lorentz transformations between two coordinate systems of the moving and rest frames. However, when we try to compare the sizes of the object and its image, the absolute times should be time-matched as shown in Fig. 2. The time-matching of the absolute times means $x=\gamma x_{x}$, in Fig. 2. The time-matched sizes of the object and its image are $x^{\prime}$ and $x=\gamma x^{\prime}$, respectively, as shown in the middle case of Fig. 2. This indicates that the size of $x^{\prime}$ ( or $\Delta x^{\prime}$ ) of the moving object

\begin{tabular}{|c|c|}
\hline $\begin{array}{l}\mathrm{x}_{1} \\
\mathrm{x}^{\prime}=\mathrm{x}_{1}-\mathrm{x}_{0}=\gamma(\mathrm{x}-\mathrm{x}-\mathrm{vt}) \\
\text { Object size }(\mathrm{x}) \text { in the rest frame is } \\
\text { extended to } \mathrm{x}^{\prime} \text { in the moving frame. } \\
\mathrm{t}^{\prime}=\gamma\left(\mathrm{t}-\mathrm{vx} / \mathrm{c}^{2}\right) \text { for } \mathrm{x}^{\prime}=\mathrm{ct} \text { ' } \\
\text { Fig. 3. The flat space corresponds } \\
\text { 4-dimensional spaces is not zero a } \\
\text { times are time-matched to induce }\end{array}$ & $\begin{array}{l}\text { Moving object: } \begin{array}{l}\mathrm{y}^{\prime}=\mathrm{y} \quad \mathrm{x}^{\prime}=\mathrm{x} \\
\mathrm{z}^{\prime}=\mathrm{z} \quad \mathrm{v} \neq 0 \\
\text { By using } \\
\mathrm{x}=\mathrm{ct} \text {, and } \mathrm{x}^{\prime}=\mathrm{ct}^{\prime} \\
\mathrm{t}_{1}=\gamma \mathrm{t}, \text { and } \mathrm{t}_{1}^{\prime}=\gamma \mathrm{t}^{\prime} \\
\mathrm{x}^{\prime}=\gamma(1-\mathrm{v} / \mathrm{c}) \mathrm{x}\end{array} \\
\mathrm{x}=\gamma(1+\mathrm{v} / \mathrm{c}) \mathrm{x}^{\prime}\end{array}$ \\
\hline
\end{tabular}

is expanded to the size of $x=\gamma x^{\prime}$ ( or $\Delta x=\gamma \Delta x^{\prime}$ ) in Fig. 2. Also, by the same principle, the absolute times of the object and its image can be compared by taking the absolute time part $\left(\mathrm{t}^{\prime}\right)$ of $\mathrm{t}=\gamma\left(\mathrm{t}^{\prime}\right.$ $\left.+v x^{\prime} / c^{2}\right)$. And $t^{\prime}=\gamma t^{\prime}$ in Fig. 3.

In Fig.1, the time momentum $\left(\mathrm{p}_{\mathrm{t}}=\mathrm{E}_{0} / \mathrm{c}\right)$ is the momentum along the time axis. When the matter does not move along the space axis, it has only the rest mass energy of $E_{0}$ with the time momentum $\left(\mathrm{E}_{0} / \mathrm{c}\right)$ along the time axis. When the matter moves with the velocity of $\mathrm{v}$ along the $\mathrm{x} 1 \mathrm{x} 2 \times 3$ space axis, the non-zero space momentum of $p_{x}$ should be used as shown in Fig 3. The total energy (E) and total momentum $(\mathrm{P}=\mathrm{cE})$ are obtained from the equations of $\vec{P}=p_{x} \hat{x}+\frac{E_{0}}{c} \hat{t}$ and $\mathrm{P}^{2}=\mathrm{p}_{\mathrm{x}}{ }^{2}+$ $\mathrm{E}_{0}{ }^{2} / \mathrm{c}^{2}$. Here the three-dimensional $\mathrm{x} 1 \times 2 \times 3$ space we live on is taken as the three-dimensional $\mathrm{x} 1 \times 2 \times 3$ space with the positive time momentum in Fig. 1. Then there are momentum and energy transitions of the particle with the rest mass of $m_{0}$. For the momentum transition of the particle, $\mathrm{t}_{1}$ $=\gamma \mathrm{t}$. The momentum space can be described in the space-time coordinates of $\left(\mathrm{x} 1, \mathrm{x} 2, \mathrm{x} 3, \mathrm{ct}_{1}\right)$. And the particle shape is transformed according to the energy transition of the particle. Then the particle 
size is enlarged following the equation of $\Delta x=\gamma \Delta x^{\prime}$ in Fig. 1. From the equation of $\Delta x=\gamma \Delta x^{\prime}$ in Figs. 1, $\Delta \mathrm{x} \rightarrow$ infinite value (flat space) as v/c $\rightarrow \mathrm{c}$ (photon speed). This means that the particles become the photon when they have the photon speed of c. In other words, only the photons have the speed of $\mathrm{c}$ as the flat space.

\section{Quantum mechanics based on the 4-D Euclidean space}

It is thought from Fig. 1 that always the $\mathrm{x} 1 \mathrm{x} 2 \mathrm{x} 3$ space with the positive time momentum and its partner $\times 1 \times 2 \times 3$ space with the negative time momentum are born in the process like the big bang from the nothing with the zero energy. This pair production of the $\mathrm{x} 1 \times 2 \times 3$ space and its partner $\mathrm{x} 1 \mathrm{x} 2 \mathrm{x} 3$ space takes place on the $\mathrm{x} 0 \mathrm{y} 0 \mathrm{z} 0$ space (mother universe) with the infinite time and space scales [15]. It is assumed that the three-dimensional quantized flat $\mathrm{x} 1 \times 2 \times 3$ space has the positive time momentum (positive energy) because our universe has the positive energy. In the present work, the energy is defined as the 4-dimensional space-time volume in Table 2.

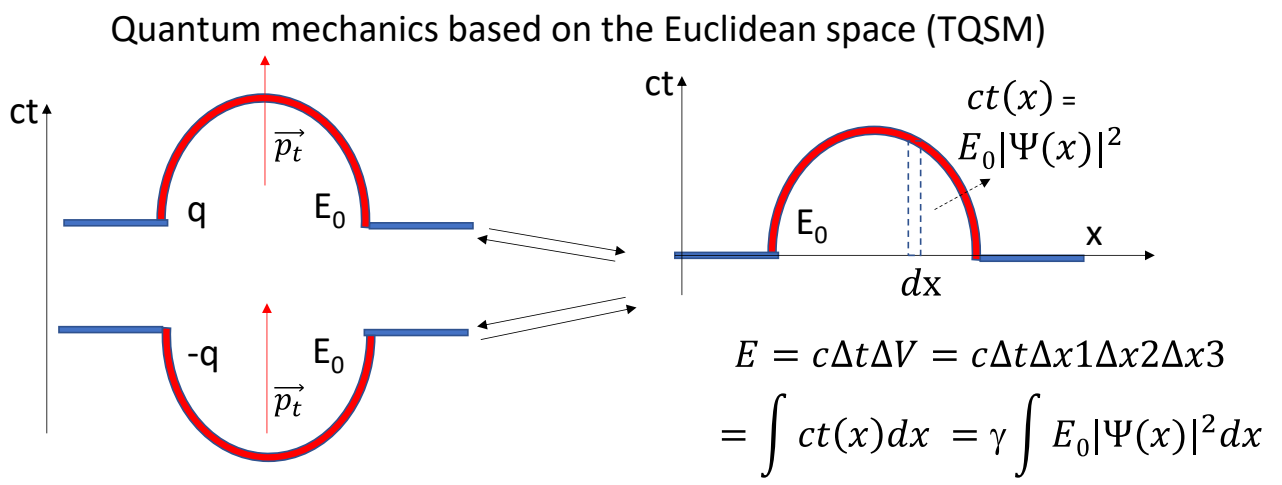

Charge conservation for the elementary particles: $|q|=c \Delta t=$ constant. The absolute time width $(c \Delta t)$ and its associated charge $(|q|=c \Delta t)$ for the elementary particles are, always, constant in all coordinate frames.

Modified Lorentz transformations for the elementary particles: $\lim _{v \rightarrow c} \Delta t \rightarrow \Delta t, \lim _{v \rightarrow c} \Delta t_{l} \rightarrow \infty, \lim _{v \rightarrow c} \Delta \mathrm{x} \rightarrow \infty, \lim _{v \rightarrow c} E \rightarrow \infty, \lim _{v \rightarrow c} \Delta V \rightarrow \infty$. $\Delta x=\gamma \Delta x_{0}, v=\Delta x / \Delta t_{1}, E=c \Delta t \Delta V=|q| \Delta V=c \Delta t \gamma \Delta V_{0}=\gamma c \Delta t \Delta V_{0}=\gamma|q| \Delta V_{0}=\gamma E_{0}$

Fig. 4. Lorentz transformations and particle wave function [16]. See Fig. 2.

Therefore, always the $\mathrm{x} 1 \times 2 \times 3$ space with the positive time displacement of $\mathrm{q}$ and the energy of $\mathrm{E}_{0}$ and its partner $\mathrm{x} 1 \times 2 \times 3$ space with the negative time displacement of $-\mathrm{q}$ and the energy of $\mathrm{E}_{0}$ are born in the process like the pair production from the $\mathrm{x} 1 \mathrm{x} 2 \times 3$ space with the energy of $2 \mathrm{E}_{0}$ in Fig. 4. This pair production of the $\mathrm{x} 1 \times 2 \times 3$ space and its partner $\times 1 \times 2 \times 3$ space takes place on the $\times 1 \times 2 \times 3$ space. The $\mathrm{x} 1 \times 2 \times 3$ flat space is warped by the time displacement. The time displacement of the flat $x 1 \times 2 \times 3$ space corresponds to the warped $x 1 \times 2 \times 3$ space in Fig. 4. The charge of the warped $\mathrm{x} 1 \mathrm{x} 2 \mathrm{x} 3$ space is defined as $|\mathrm{q}|=\mathrm{c} \Delta \mathrm{t}$ of the warped $\mathrm{x} 1 \mathrm{x} 2 \mathrm{x} 3$ space [16] which is called as the electric charge. The warped $\times 1 \times 2 \times 3$ space with the positive charge of $+q>0$ is defined as the anti-matter and the partner $\times 1 \times 2 \times 3$ space with the negative charge of $-\mathrm{q}<0$ is defined as the matter. The total time displacement of the warped $\times 1 \times 2 \times 3$ space is the 4-dimensional space-time volume called as the energy $\left(E=E_{0}\right)$ of the warped $x 1 \times 2 \times 3$ space in Table 2 and Fig. 4 [16]. The flat space has the zero charge which means the zero warped space-time volume. The warped space of the particle 
can be explained as the wave function of the quantum mechanics in Fig. 4. The size of the particle follows the Modified Lorentz transformations in Fig. 1.

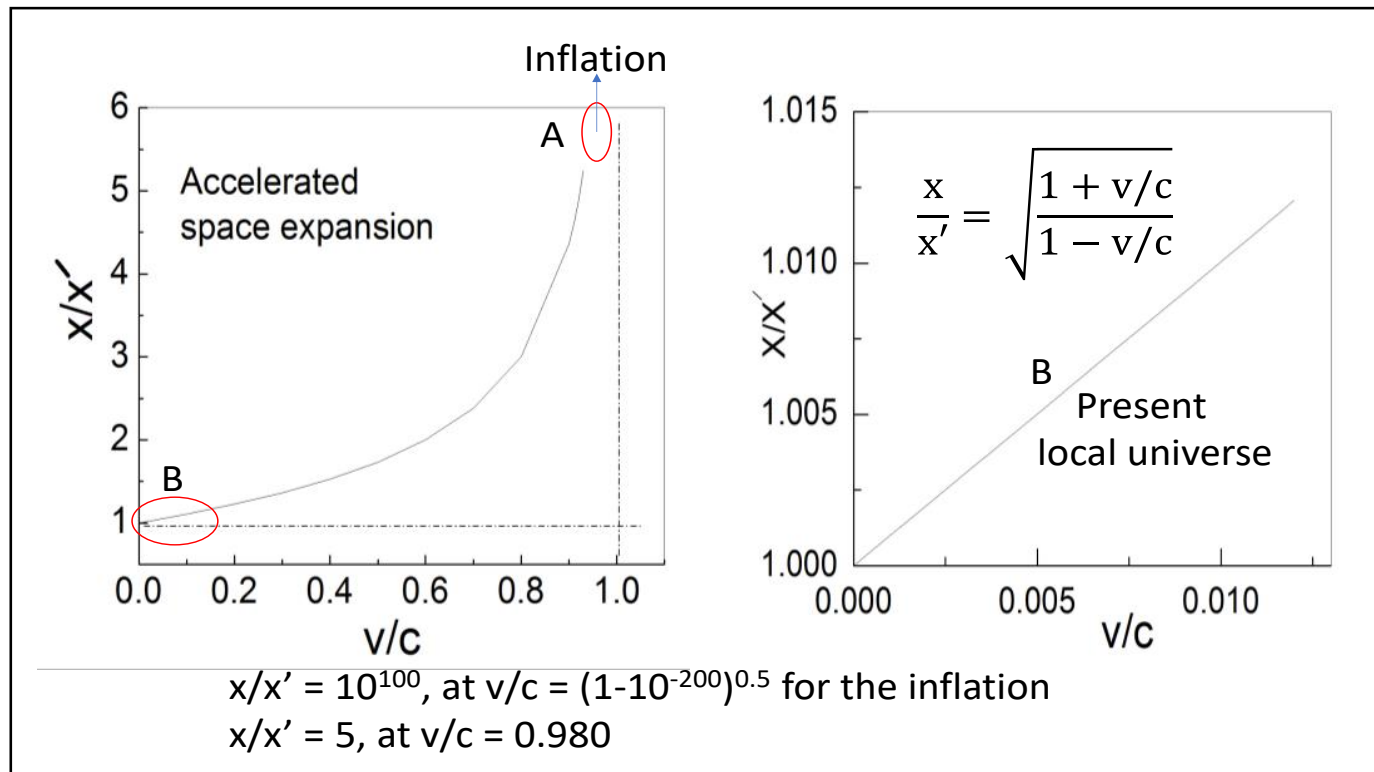

Fig. 5 The accelerated expansion and inflation of the universe are explained. See Figs. 2 and 3.

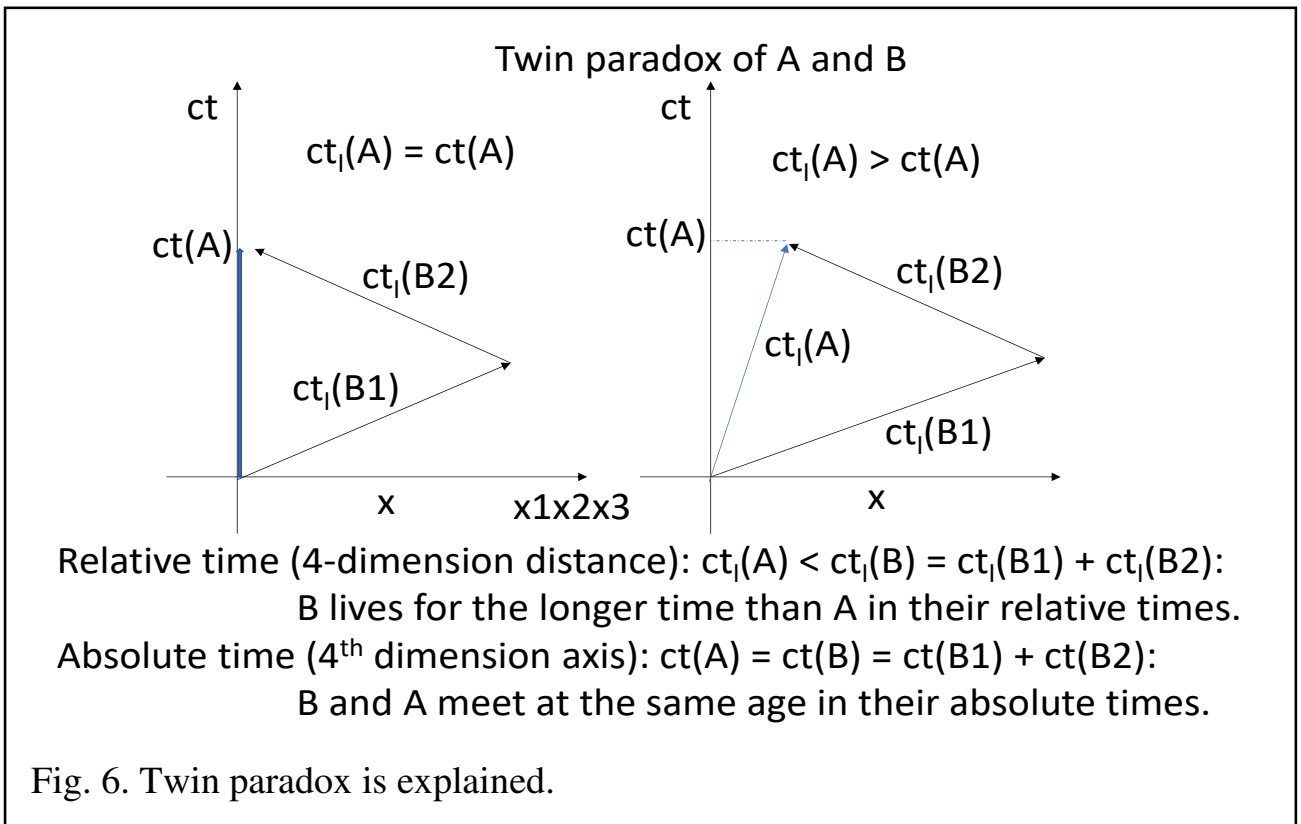

In Fig. 5, It has been known that the inflation could be explained based on the modified Lorentz transformations. If the universe at the big bang has the very fast speed as shown in Fig. 5, the universe can experience the inflation of the sudden size expansion to the huge size. Current space expansion of our universe could be related to the space size expansion due to the Modified Lorentz transformations in Figs. 2 and 3. 
The elementary particles have the three kinds of charges of the electric charges (EC), lepton charges (LC) and color charges (CC) $[11.15,16]$. In the present work, each charge corresponds to one three-dimensional quantized warped space $[11,15,16]$. So, the warped $\times 1 \times 2 \times 3$ space corresponds to the electric charge. It is proposed that the second warped $\times 4 \times 5 \times 6$ space and the third warped $\times 7 \times 8 \times 9$ space correspond to the lepton charge and color charge, respectively. The leptons with the electric charges and lepton charges are originated from the intertwined states of the warped $\times 1 \times 2 \times 3$ and $\times 4 \times 5 \times 6$ spaces. The quarks with the electric charges, lepton charges and color charges are originated from the intertwined states of the warped $\times 1 \times 2 \times 3, \times 4 \times 5 \times 6$ and $\times 7 \times 8 \times 9$

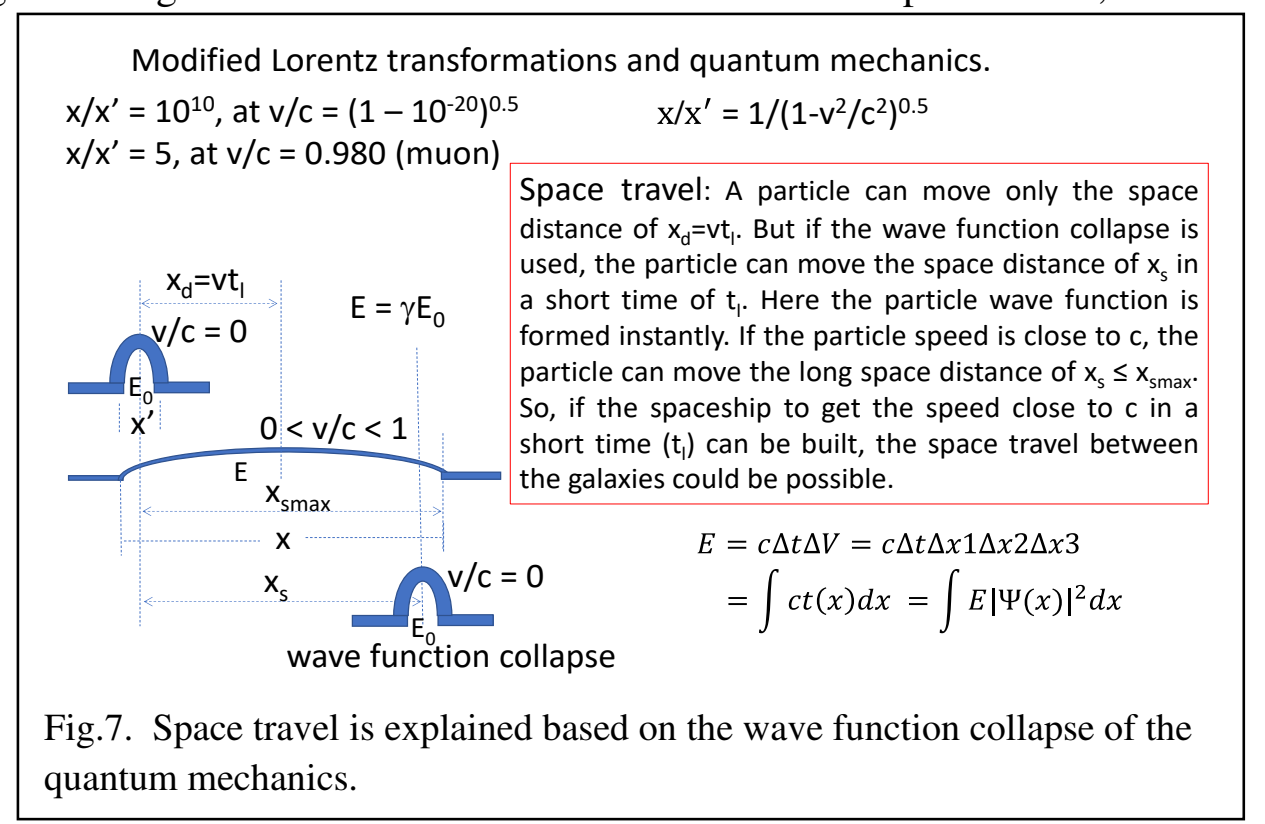

spaces. In Table 2, the simple comparison between the quantum mechanics and general relativity is shown for the short reviewing of the volume and distance of the 4-dimensional space and time. In Fig. 3, it is indicated that the Modified Lorentz transformations are based on the flat photon space.

4. Absolute time, twin paradox, space travel and instant force action

In the 4-dimension coordinate system, the $4^{\text {th }}$ dimension axis is the absolute time $(\mathrm{t})$ axis in the present work as shown in Figs. 1-4. The physical space of our universe is based on the flat space. Then the flat space corresponds to the photon space. Therefore, the flat space is moving with the light speed of $\mathrm{c}[15,16]$. The absolute time can be expressed as ct in the 4-dimension coordinate system. Then the twin paradox of two persons (A and B) is described in Fig. 6. Two persons meet at the same starting position of the earth. Then in the left figure, the A person is just at the same position of the earth and the B person travels to the star, comes back to the earth and meets the A person at the earth. In the right figure, the A and B persons are traveling by using the different spaceships. And two persons meet at the star. Then the twin paradox is whether two persons are at the same age or not. This looks like the 4-dimensional vector addition problem. The 4-dimension distance corresponds to the traveling relative time of $\mathrm{ct}_{\mathrm{l}}$. The relative time (4-dimension distance) is $\operatorname{ct} 1(\mathrm{~A})<\operatorname{ct}_{1}(\mathrm{~B})=\operatorname{ct}_{1}(\mathrm{~B} 1)+\operatorname{ct}(\mathrm{B} 2)$ : The B person lives for the longer time than the A person in their relative times. And the $4^{\text {th }}$ dimension coordinate is the absolute time of ct. Then the absolute 
time is $\operatorname{ct}(\mathrm{A})=\operatorname{ct}(\mathrm{B})=\operatorname{ct}(\mathrm{B} 1)+\operatorname{ct}(\mathrm{B} 2)$. Therefore, two persons of $\mathrm{B}$ and $\mathrm{A}$ meet at the same age in their absolute times. Two persons lives the same age if the same absolute time clocks are used for the $\mathrm{A}$ and $\mathrm{B}$ persons. The twin paradox takes place because the A and B persons use the different relative time clocks.

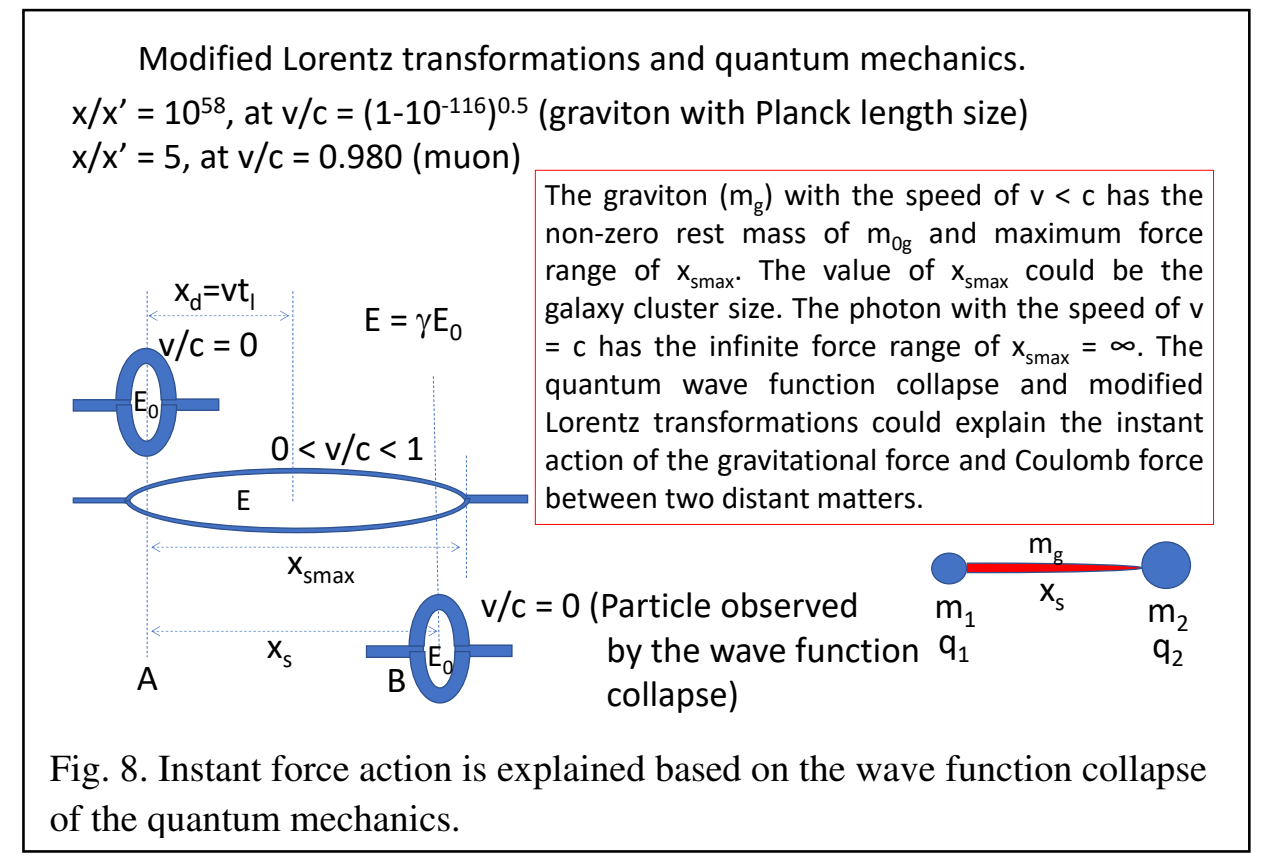

In Fig. 7, the space travel could be possible by using the wave function collapse and Modified Lorentz transformations. Also, in Fig. 8, the instant action of the gravitational force and Coulomb force can be explained by using the wave function collapse and Modified Lorentz transformations. These two cases are shown as two examples to which the Modified Lorentz transformations can be applied.

\section{Summary}

In summary, the Lorentz transformations in the special relativity are modified based on the 4-D Euclidean space rather than 4-D Minkowski space in the present work. The $4^{\text {th }}$ dimension is assigned as the real absolute time (ct) axis and energy axis $\left(\mathrm{cP}_{\mathrm{t}}=\mathrm{E}_{0}\right)$. This $4^{\text {th }}$ dimension can be indirectly felt through the observable relative time $\left(\mathrm{ct}_{\mathrm{l}}\right)$ and observable total energy $\left(\mathrm{cP}_{\mathrm{tl}}=\mathrm{E}\right)$. The space-time distance is $\mathrm{d}(\mathrm{x} 1 \times 2 \times 3 \times 4)=\mathrm{ct}_{\mathrm{t}}$. The relative time of $\mathrm{t}$ in the special relativity based on 4D Minkowski space is expressed as the relative time of $t_{1}$ in the Modified Lorentz transformations based on the 4-D Eulcidean space of the present paper. The modified Lorentz transformations are introduced by using the present space-time geometry. In Figs. 2 and 3, the final modified Lorentz transformations can be used generally to any length of the flat spaces. It is concluded that the special and general relativities can be modified based on this modified Lorentz transformations. These modified Lorentz transformations are approximated to the Lorentz transformations as $t \rightarrow$ $\mathrm{t}_{\mathrm{l}}$ when $\mathrm{v} / \mathrm{c}<<1$ and to the Galilean transformations as $\mathrm{v} / \mathrm{c}$ is close to zero in Table 1 . In the present work, it is proposed that the accelerated space expansion of the universe can be explained in terms of the modified Lorentz transformations. 
When we try to compare the sizes of the object and its image, the absolute times should be timematched. The time-matching of the absolute times means $x=\gamma x_{x}$, in Fig. 2. The time-matched sizes of the object and its image are $x^{\prime}$ and $x=\gamma x^{\prime}$, respectively, as shown in the middle case of Fig. 2. This indicates that the size of $\mathrm{x}^{\prime}$ ( or $\Delta \mathrm{x}^{\prime}$ ) of the moving object is expanded to the size of $\mathrm{x}$ $=\gamma x^{\prime}\left(\right.$ or $\left.\Delta x=\gamma \Delta x^{\prime}\right)$ in Fig. 2.

The twin paradox is explained in terms of the 4-dimension coordinate system. The twin paradox is caused by using the different relative time clocks observing the 4-dimension distance of ct. If two persons use the same absolute time clocks observing the $4^{\text {th }}$ dimension coordinate of ct, there is no twin paradox. The relative time $\left(\mathrm{t}_{\mathrm{l}}\right)$ and energy $(\mathrm{E})$ are defined as the 4-dimensional distance and 4-dimensional volume, respectively. The geometrical space-time shape has the (x1,x2,x3,ct) coordinate system with the metric signature of $(++++)$ but not the $\left(x 1, x 2, x 3, c_{1}\right)$ coordinate system with the metric signature of $(+--)$. Therefore, $\mathrm{d}(\mathrm{x} 1 \times 2 \times 3 \times 4)^{2}=\left(\mathrm{ct}_{1}\right)^{2}=(\mathrm{ct})^{2}+\mathrm{x}^{2}=\mathrm{x} 1^{2}+$ $\mathrm{x} 2^{2}+\mathrm{x} 3^{2}+\mathrm{x} 4^{2}$ and $\mathrm{V}(\mathrm{x} 1 \mathrm{x} 2 \mathrm{x} 3 \mathrm{x} 4)=\mathrm{E}=\mathrm{mc}^{2}=\Delta(\mathrm{ct}) \Delta \mathrm{x} 1 \Delta \mathrm{x} 2 \Delta \mathrm{x} 3$ from $(\mathrm{x} 1, \mathrm{x} 2, \mathrm{x} 3, \mathrm{x} 4)$ of the geometrical space-time shape.

Finally, the wave functions and energy of the moving particle are described in terms of the present modified Lorentz transformations. In this physical concept, the space travel between the galaxies could be possible if the spaceship with the velocity close to the photon velocity of can be built in the future. Also, it is shown that the quantum wave function collapse and modified Lorentz transformations could explain the instant action of the gravitational force and Coulomb force between two distant matters.

\section{References}

[1] Albert Einstein, Relativity: The Special and General Theory (Henry holt and company, New york 1921).

[2] Max Born, Einstein's Theory of Relativity (Courier Dover Pub,. 2012) pp. 236-237.

[3] E.T. Kipreos and R.S. Balachandran, Mod. Phys. Lett. A31, 1650157 (2016).

[4] V.A. Kostelecky and M. Mewes, Phys. Rev. D66, 056005 (2002).

[5] D. Mattingly, Living Rev. Relativ. (2005) 8: 5. https://doi.org/10.12942/lrr-2005-5

[6] L. Boyle, K. Finn and N. Turok, Phys. Rev. Lett. 121, 251301 (2018).

[7] R.M. Wald, Phys. Rev. D21, 2742 (1980).

[8] K. Svozil, Europhys. Lett. 2, 83 (1986).

[9] K. Rebilas, American J. of Phys. 78, 294 (2010).

[10] R. Potting, J. of Phys.: Conf. Ser. 447, 012009 (2013).

[11] Jae-Kwang Hwang, Mod. Phys. Lett. A32, 1730023 (2017).

[12] A. Kostelecky and A.J. Vargas, Phys. Rev. D98, 036003 (2018).

[13] V.A. Kostelecky, Phys. Rev. D69, 105009 (2004).

[14] C.N. Cruz et al., Int. J. Mod. Phys. D27, 1850011 (2018).

[15] Jae-Kwang Hwang, Preprints 2020, 2020080726 (doi: 10.20944/preprints202008.0726.v1).

[16] Jae-Kwang Hwang, Preprints 2021, 2021020392 (doi: 10.20944/preprints202102.0392.v1). 


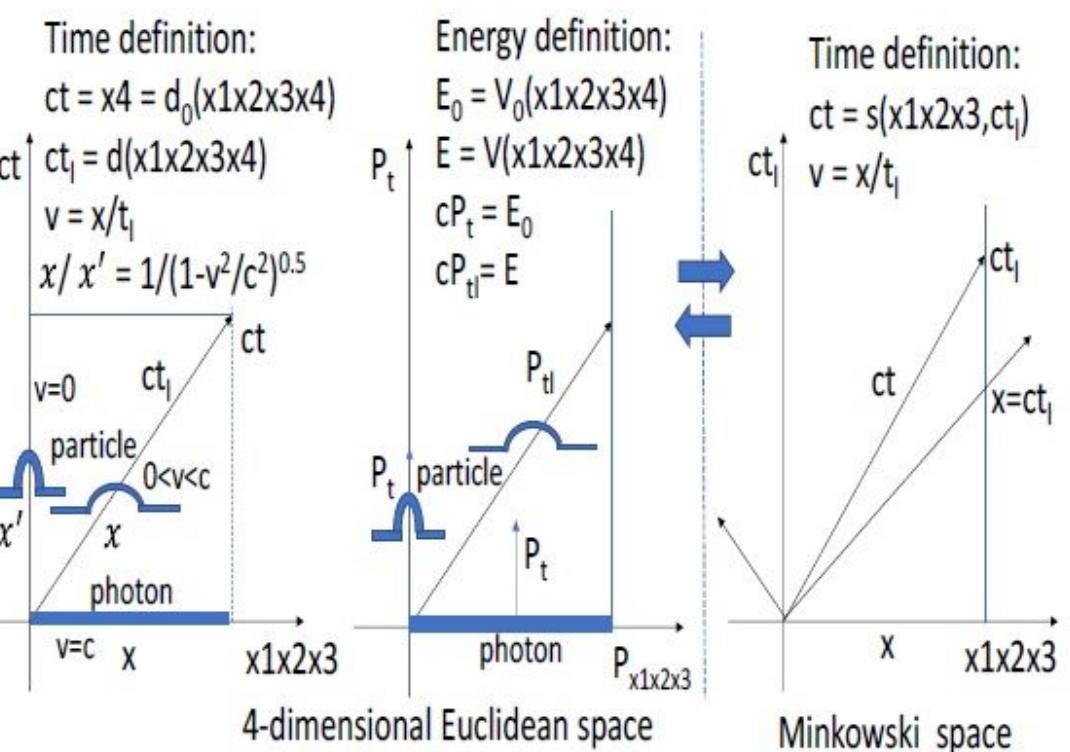

We live in the $\times 1 \times 2 \times 3$ space. The $4^{\text {th }}$ dimension can be indirectly felt through the observation of the relative time $\left(c_{t}\right)$ and energy $\left(\mathrm{CP}_{\mathrm{t}}\right)$. The space-time distance is $d(\times 1 \times 2 \times 3 \times 4)=c t_{1}$. The rest mass $\left(m_{0}\right)$ is defined as $m_{0}=E_{0} / c^{2}$.
$E=m c^{2}, E_{0}=m_{0} c^{2}, t_{1}=\gamma t$, t: absolute time, $t_{\text {; }}$ relative time

$p_{t}=m_{0} c \frac{x 4}{c t}=\frac{\gamma E_{0}}{c^{2}} \frac{x 4}{\gamma t}=\frac{E x 4}{c t_{l}}=\frac{E x 4}{c d}=m c \frac{x 4}{c t_{l}}=m \frac{x 4}{t_{l}}=\frac{E_{0}}{c}$

$p_{x}=m_{0} c \frac{x}{c t}=\frac{\gamma E_{0}}{c^{2}} \frac{x}{\gamma t}=\frac{E}{c c t_{l}}=\frac{x}{c d}=m c \frac{x}{c t_{l}}=m v=\frac{E}{c c}$

$p_{t l}=m_{0} c \frac{d}{c t}=\frac{\gamma E_{0}}{c^{2}} \frac{d}{\gamma t}=\frac{E}{c c t_{l}}=m c \frac{d}{c t_{l}}=m c=\frac{E}{c}$

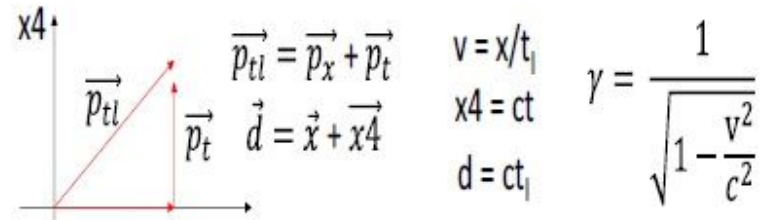

$\overrightarrow{p_{x}} \quad \times 1 \times 2 \times 3$

Note that the negative time, negative mass (energy) and negative momenta are possible. $E_{0}<0$ and $m_{0}<0$ for $p_{t}<0$.

\section{Figure 1}

Time and energy are defined. See Figs. 2 and 3.

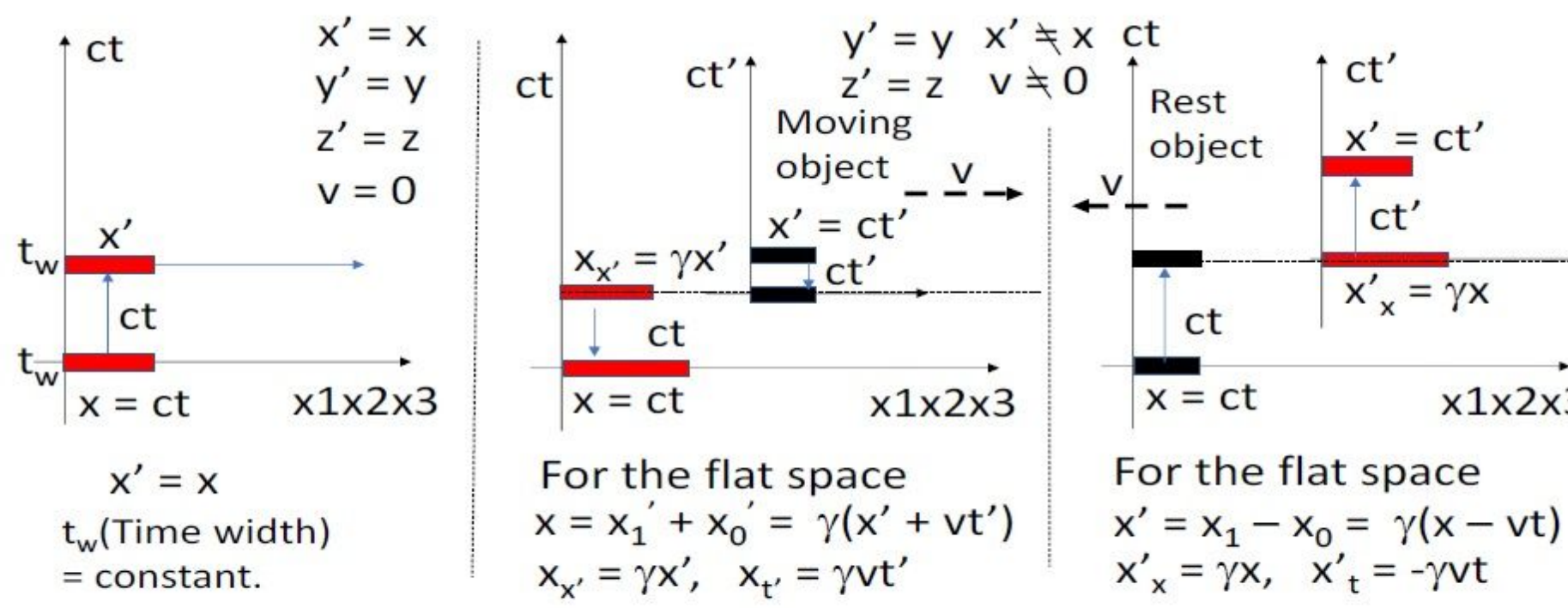

Object ( $(-)$ ) and its image should be time-matched by the absolute times of $\mathrm{x}=\mathrm{ct}$ and $\mathrm{x}^{\prime}=\mathrm{ct}^{\prime}$ when they are compared in the rest and moving frames.

The time matched size is $\Delta x=x=x_{x^{\prime}}=\gamma x^{\prime}=\gamma \Delta x^{\prime}$ to show the expansion of the size.

Figure 2 
The whole flat space treated like a rest particle is shown in the 4-dimensional space. The flat space corresponds to the photons. See Fig. 3.

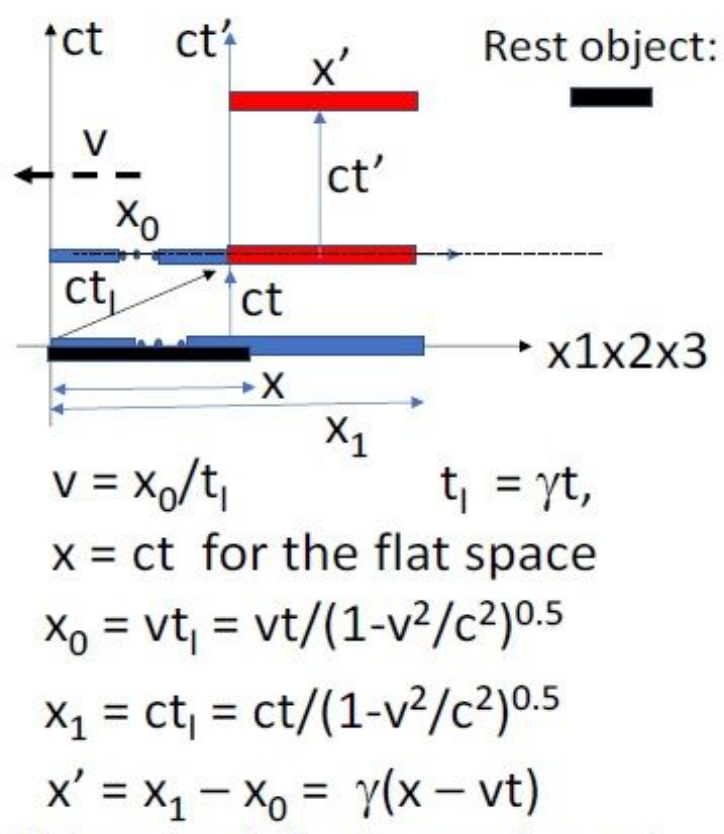

Object size $(x)$ in the rest frame is extended to $x^{\prime}$ in the moving frame. $t^{\prime}=\gamma\left(t-v x / c^{2}\right)$ for $x^{\prime}=c^{\prime}$
Moving object:

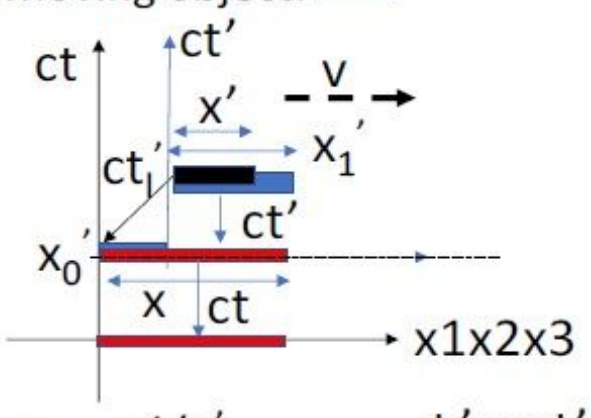

$$
y^{\prime}=y \quad x^{\prime} \neq x
$$$$
z^{\prime}=z \quad v \neq 0
$$

By using

$$
\mathrm{x}=\mathrm{ct} \text {, and } \mathrm{x}^{\prime}=\mathrm{ct}^{\prime}
$$$$
\mathrm{t}_{1}=\gamma \mathrm{t} \text {, and } \mathrm{t}_{1}{ }^{\prime}=\gamma \mathrm{t}^{\prime}
$$$$
\mathrm{x}^{\prime}=\gamma(1-\mathrm{v} / \mathrm{c}) \mathrm{x}
$$

$$
v=x_{0}{ }^{\prime} / t_{1}^{\prime} \quad t_{1}^{\prime}=\gamma t^{\prime}
$$$$
\mathrm{x}=\gamma(1+\mathrm{v} / \mathrm{c}) \mathrm{x}^{\prime}
$$

$\mathrm{x}^{\prime}=\mathrm{ct}^{\prime}$ for the flat space

$\mathrm{x}_{0}^{\prime}=\mathrm{vt}_{1}^{\prime}=\mathrm{vt}^{\prime} /\left(1-\mathrm{v}^{2} / \mathrm{c}^{2}\right)^{0.5}$

$\mathrm{t}^{\prime}=\gamma(1-\mathrm{v} / \mathrm{c}) \mathrm{t}$

$\mathrm{t}=\gamma(1+\mathrm{v} / \mathrm{c}) \mathrm{t}^{\prime}$

$\mathrm{x}_{1}{ }^{\prime}=\mathrm{ct}_{1}^{\prime}=\mathrm{ct}^{\prime} /\left(1-\mathrm{v}^{2} / \mathrm{c}^{2}\right)^{0.5}$

$\mathrm{t}_{1}^{\prime}=\gamma(1-\mathrm{v} / \mathrm{c}) \mathrm{t}_{1}$

$\mathrm{x}=\mathrm{x}_{1}{ }^{\prime}+\mathrm{x}_{0}{ }^{\prime}=\gamma\left(\mathrm{x}^{\prime}+\mathrm{vt^{ \prime }}\right)$

Object size $\left(x^{\prime}\right)$ in the moving frame is

extended to $x$ in the rest frame.

$$
t=\gamma\left(t^{\prime}+v x^{\prime} / c^{2}\right) \text { for } x=c t
$$

\section{Figure 3}

The flat space corresponds to the photons. Here, the relative velocity ( $v$ ) of two 4-dimensional spaces is not zero and along the $\mathrm{x}$ axis with $\mathrm{v}-\mathrm{x} 0 / \mathrm{t} 1$. The absolute times are time-matched to induce the modified Lorentz transformations . 
Quantum mechanics based on the Euclidean space (TQSM)
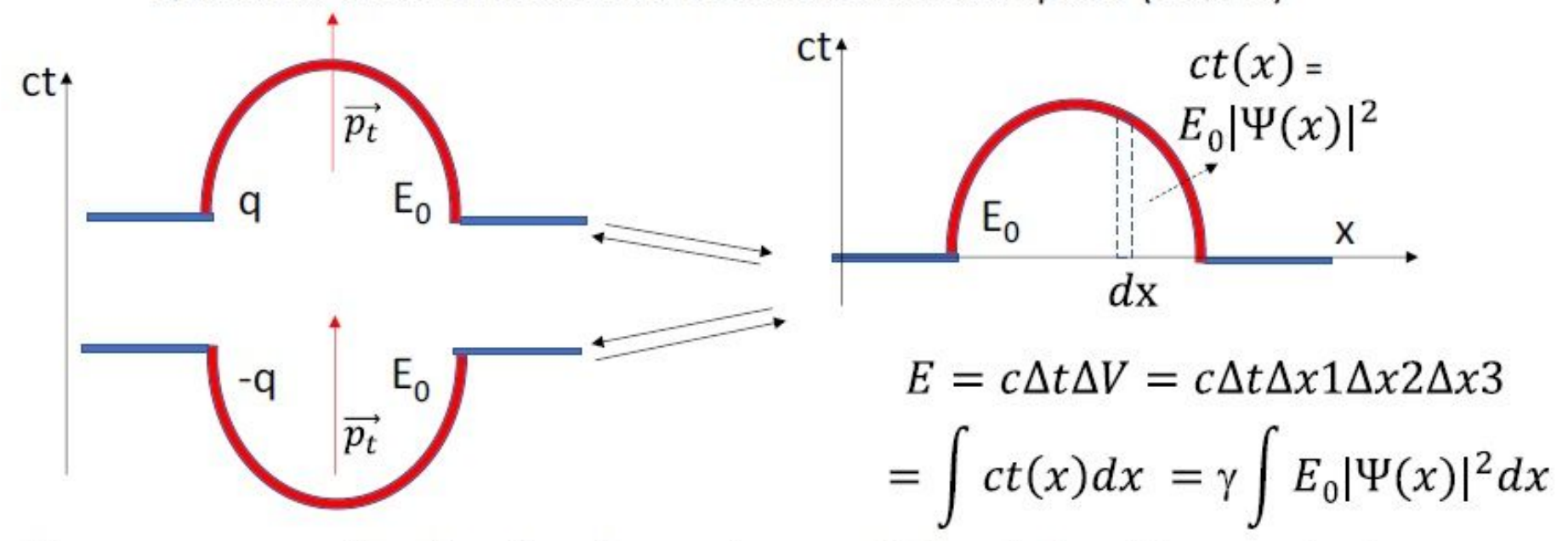

Charge conservation for the elementary particles: $|q|=c \Delta t=$ constant. The absolute time width $(\mathrm{c} \Delta \mathrm{t})$ and its associated charge $(|\mathrm{q}|=\mathrm{c} \Delta \mathrm{t})$ for the elementary particles are, always, constant in all coordinate frames.

Modified Lorentz transformations for the elementary particles:

$\lim _{v \rightarrow c} \Delta t \rightarrow \Delta t, \lim _{v \rightarrow c} \Delta t_{l} \rightarrow \infty, \lim _{v \rightarrow c} \Delta \mathrm{x} \rightarrow \infty, \lim _{v \rightarrow c} E \rightarrow \infty, \lim _{v \rightarrow c} \Delta V \rightarrow \infty$.

$\Delta \mathrm{x}=\gamma \Delta \mathrm{x}_{0}, \mathrm{v}=\Delta \mathrm{x} / \Delta \mathrm{t}_{\mathrm{l}}, \mathrm{E}=\mathrm{c} \Delta \mathrm{t} \Delta \mathrm{V}=|\mathrm{q}| \Delta \mathrm{V}=\mathrm{c} \Delta \mathrm{t} \gamma \Delta \mathrm{V}_{0}=\gamma \mathrm{c} \Delta \mathrm{t} \Delta \mathrm{V}_{0}=\gamma|\mathrm{q}| \Delta \mathrm{V}_{0}=\gamma \mathrm{E}_{0}$

\section{Figure 4}

Lorentz transformations and particle wave functions [16]. See Fig. 2.

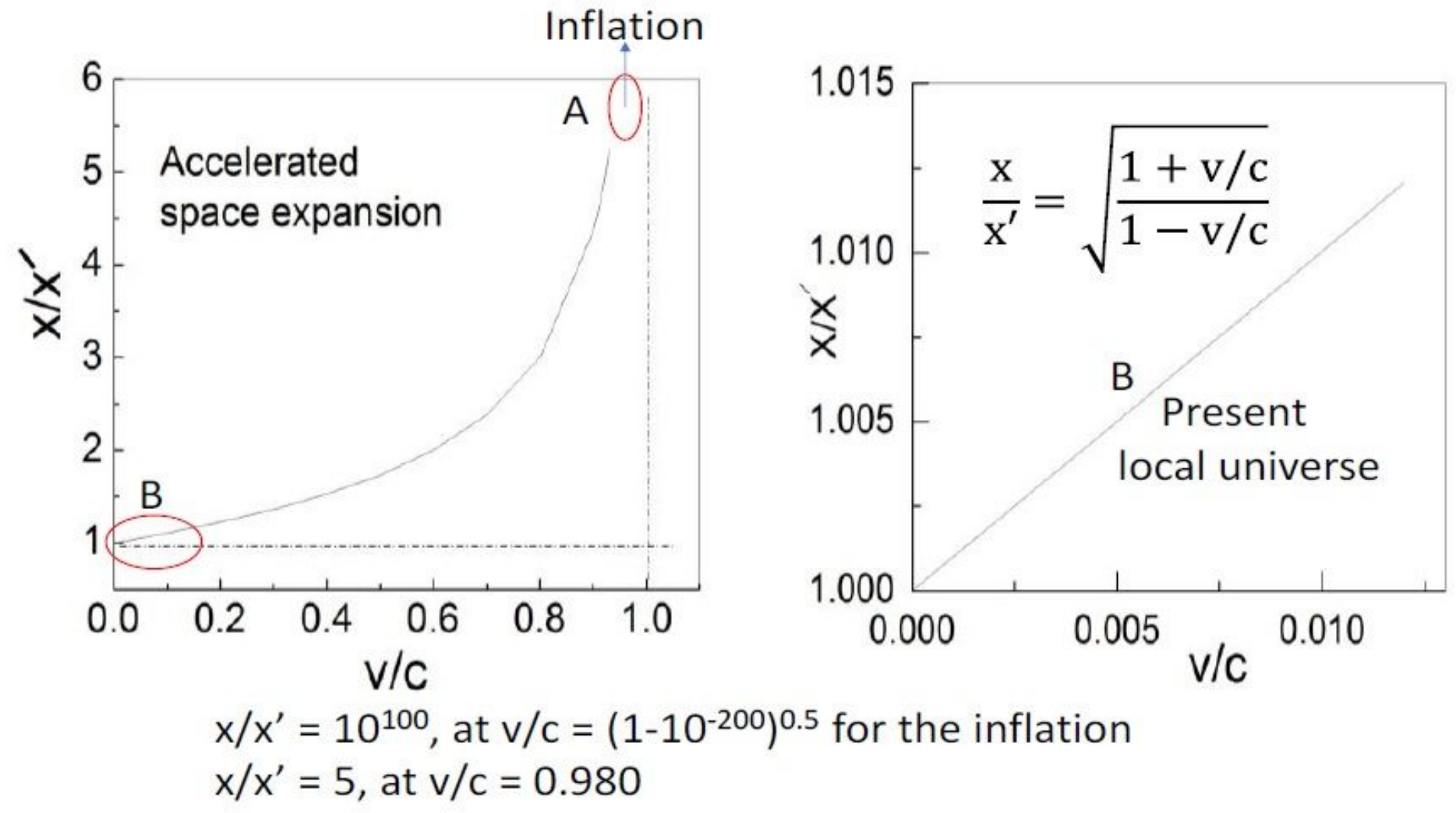


Figure 5

The accelerated expansion and inflation of the universe are explained. See Figs. 2 and 3.

Twin paradox of $A$ and $B$
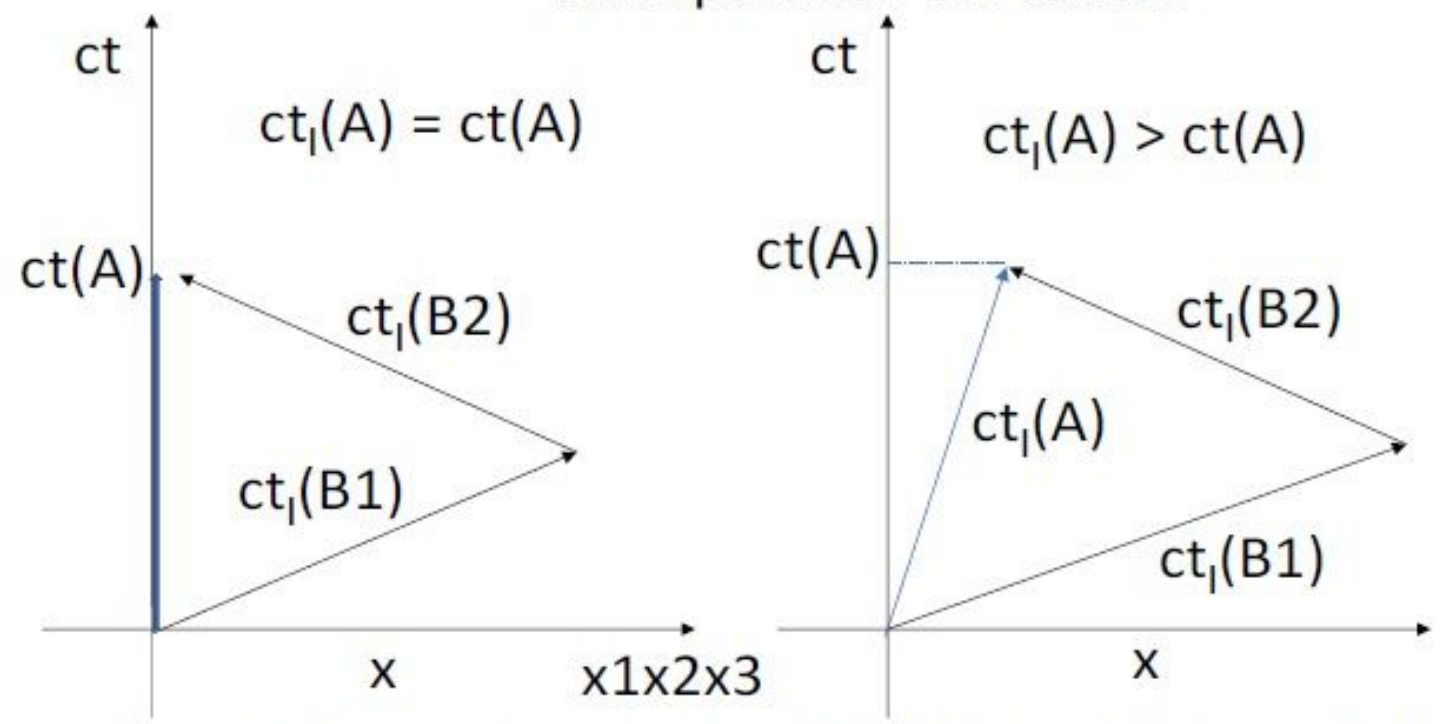

Relative time (4-dimension distance): $\mathrm{ct}_{1}(\mathrm{~A})<\mathrm{ct}_{1}(\mathrm{~B})=\mathrm{ct}_{1}(\mathrm{~B} 1)+\mathrm{ct}_{1}(\mathrm{~B} 2)$ :

$B$ lives for the longer time than $A$ in their relative times.

Absolute time ( $4^{\text {th }}$ dimension axis): $\operatorname{ct}(A)=\operatorname{ct}(B)=\operatorname{ct}(B 1)+\operatorname{ct}(B 2)$ :

$B$ and $A$ meet at the same age in their absolute times.

Figure 6

Twin paradox is explained. 
Modified Lorentz transformations and quantum mechanics.

$$
\begin{aligned}
& x / x^{\prime}=10^{10}, \text { at } v / c=\left(1-10^{-20}\right)^{0.5} \quad x / x^{\prime}=1 /\left(1-v^{2} / c^{2}\right)^{0.5} \\
& x / x^{\prime}=5, \text { at } v / c=0.980 \text { (muon) }
\end{aligned}
$$

Space travel: A particle can move only the space distance of $\mathrm{x}_{\mathrm{d}}=\mathrm{vt}$. But if the wave function collapse is

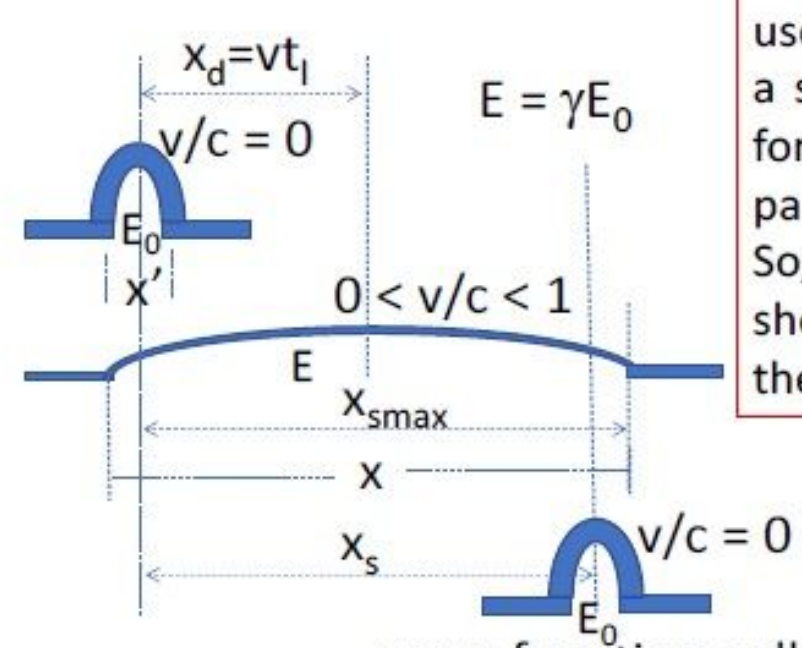
used, the particle can move the space distance of $x_{s}$ in a short time of $t_{1}$. Here the particle wave function is formed instantly. If the particle speed is close to $c$, the particle can move the long space distance of $x_{5} \leq x_{s \max }$. So, if the spaceship to get the speed close to $c$ in a short time $\left(\mathrm{t}_{1}\right)$ can be built, the space travel between the galaxies could be possible.

$$
\begin{aligned}
E & =c \Delta t \Delta V=c \Delta t \Delta x 1 \Delta x 2 \Delta x 3 \\
& =\int c t(x) d x=\int E|\Psi(x)|^{2} d x
\end{aligned}
$$

wave function collapse

Figure 7

Space travel is explained based on the wave function collapse of the quantum mechanics.

Modified Lorentz transformations and quantum mechanics.

$\mathrm{x} / \mathrm{x}^{\prime}=10^{58}$, at $\mathrm{v} / \mathrm{c}=\left(1-10^{-116}\right)^{0.5}$ (graviton with Planck length size)

$\mathrm{x} / \mathrm{x}^{\prime}=5$, at $\mathrm{v} / \mathrm{c}=0.980$ (muon)

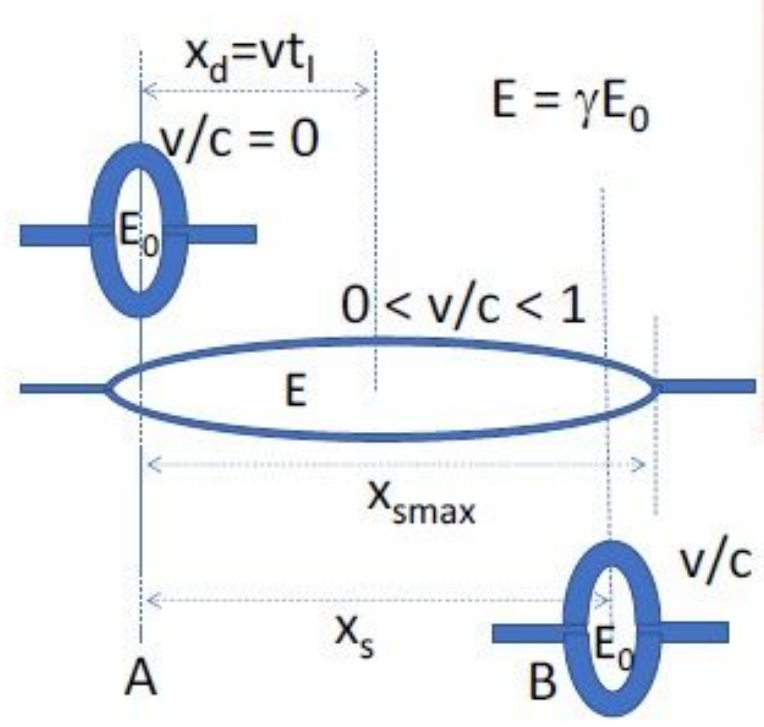

The graviton $\left(m_{g}\right)$ with the speed of $v<c$ has the non-zero rest mass of $\mathrm{m}_{0 \mathrm{~g}}$ and maximum force range of $x_{\text {smax }}$. The value of $x_{\text {smax }}$ could be the galaxy cluster size. The photon with the speed of $v$ $=c$ has the infinite force range of $x_{\text {smax }}=\infty$. The quantum wave function collapse and modified Lorentz transformations could explain the instant action of the gravitational force and Coulomb force between two distant matters.

$$
\begin{array}{r}
v / c=0 \text { (Particle observed } \quad m_{1} \\
\text { by the wave function } q_{1}
\end{array}
$$$$
\mathrm{m}_{\mathrm{g}}
$$

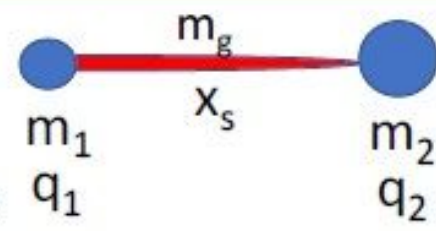
collapse) 
Figure 8

Instant force action is explained based on the wave function collapse of the quantum mechanics. 